\title{
Characterization of the $\beta$-barrel Assembly Machinery in Nanodiscs using Cryo-EM
}

\author{
$\underline{\text { Runrun }} \mathbf{W u}^{\mathbf{a}}$ and Nicholas Noinaj*, a \\ ${ }^{a}$ Markey Center for Structural Biology, Department of Biological Sciences, Purdue University, \\ West Lafayette, IN 47907, USA \\ *E-mail:nnoinaj@purdue.edu
}

\begin{abstract}
The $\beta$-barrel assembly machinery (BAM) is a $200 \mathrm{kDa}$ five component complex within the outer membrane of Gram-negative bacteria. BAM is responsible for the biogenesis of outer membrane proteins (OMPs), which are essential for nutrient import, signaling, and adhesion. The structure of BAM was first solved by crystallography [1], which demonstrated the opening of the exit pore and rearrangement at the lateral gate in the periplasmic domain of BamA, the central component for BAM. Based on these reports, it was tempting to propose that the BamB component of BAM may be regulating its conformational states. Later, the lateral gate opening was also observed in the cryo-EM structure, which was contrary to BamB's suggested role in regulating BAM's conformational states. However, one caution is that all the reported structures were solved in detergents rather than within a lipid bilayer. Therefore, in order to determine the native state of BAM within a membrane bilayer, we determined the cryo-EM structure of BAM in nanodiscs of varying sizes. In our $3 \mathrm{D}$ reconstruction, we observe BAM in the outward-open state in which the barrel of BamA, the central and essential component, is open towards the surface and closed along the periplasmic face (Fig. 1). We propose that our structure represents the true active state of BAM found within bacteria.
\end{abstract}
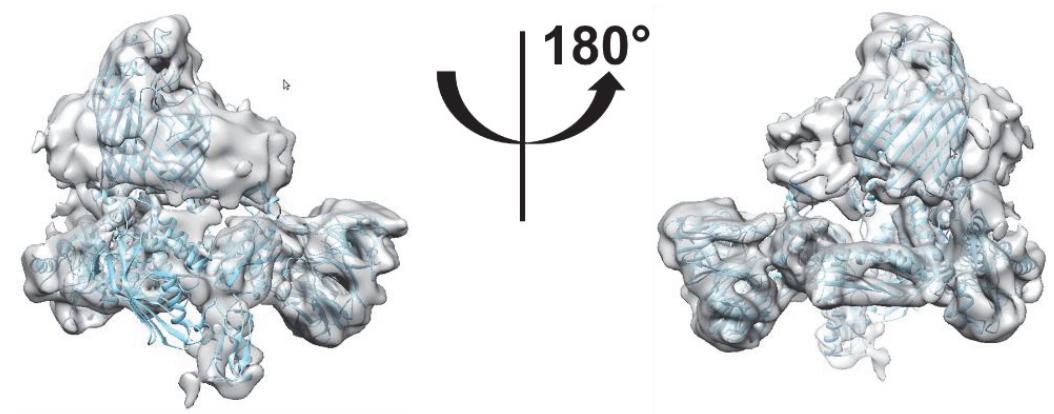

Fig. 1 Cryo-EM structure of BAM in nanodisc.

\section{$\underline{\text { References }}$}

[1] Bakelar, J., Buchanan, S. K., \& Noinaj, N. (2016). Science, 351(6269), 180-186. 